\title{
Measuring Self-Serving Cognitive Distortions: An analysis of the Psychometric Properties of the How I think Questionnaire (HIT-16-Q)
}

\author{
Eshrat Ara $^{1 *}$
}

\section{ABSTRACT}

The How I Think Questionnaire (HIT-Q; Barriga et al., 2001) is a self-report measure of selfserving cognitive distortions. This study aimed to analyze the psychometric properties of the validated version HIT-16-Q (Ara \& Shah, 2015) scores in large sample adolescents. Results showed good reliability of the total HIT-16-Q scores: alpha .83. Exploratory Factor Analysis (EFA) revealed a single factor. Confirmatory factor analysis (CFA), revealed the better fit for the one-dimensional structural model. Moreover, the HIT-16-Q has good convergent validity.

Keywords: Reliability; Validity; Factor analysis; Cognitive distortions; HIT-16-Q.

Cognitive distortions are erroneous or biased ways of attending to or applying meaning to everyday situations (Barriga, Gibbs, Potter, \& Liau, 2001). By serving to protect the individual from self-blame and a negative self-concept, self-serving cognitive distortions lessen inhibitions and permit an individual to justify antisocial behaviour. For several years, cognitive distortions have been the focus of research and intervention studies related to various types of antisocial behaviour and different youth populations.

The How I Think Questionnaire (HIT-Q; Barriga et al., 2001) was designed to measure four categories of self-serving cognitive distortions: (i) Self-Centred that is according status to one's own views, needs, immediate feelings, and desires to such a degree that the legitimate views, etc., of others are scarcely considered or are disregarded altogether; (ii) Blaming Others, that is misattributing blame to outside sources, especially another person or a momentary aberration or misattributing blame for one's victimization or other misfortune to innocent others; (iii) Minimizing/Mislabelling, that is depicting antisocial behaviour as causing no real harm or as being acceptable, or referring to others with a dehumanizing label; and (iv) Assuming the Worst: Gratuitously attributing hostile intentions to others, considering a worst-case scenario for a social situation as if it was inevitable, or assuming that improvement is impossible in one's own or other’s behaviour (Barriga et al., 20001; Barriga et al. 20008).

\footnotetext{
${ }^{1}$ Department of Psychology, University of Kashmir, J\&K, India *Corresponding Author

(C) 2015 I E Ara; licensee IJIP. This is an Open Access Research distributed under the terms of the Creative Commons Attribution License (http://creativecommons.org/licenses/by/2.0), which permits unrestricted use, distribution, and reproduction in any Medium, provided the original work is properly cited.
} 
The HIT-Q is a 54-item measure and requires subjects to rate their responses on a Likert scale ranging from "Agree strongly" (scored as 6) to "Disagree strongly" (scored as 1). Thirty-nine of the items state self-serving cognitive distortions and are divided into the four categories. Eight items constitute the Anomalous Responding (AR) scale and are included to detect insincere or otherwise suspect responding. If a protocol produces an AR mean score of 4.25 or above, the protocol is considered invalid. The seven remaining items are non-scored "positive fillers". For a more detailed description of the questionnaire see Barriga et al. (2001).

The HIT-Q is increasingly used across different disciplines both for research purposes and to assess changes after interventions and educational programmes, such as the EQUIP Programme and the Aggression Replacement Training (e.g., van der Velden, Brugman, Boom, \& Koops, 2010). The original English version has been translated and adapted in different languages, including Dutch, French, Spanish, and Swedish (Plante, Daigle, Gaumont, Charbonneau, Gibbs, \& Barriga, 2012; Rahim, Syariani, Azizah, \& Ayu, 2013). As for the psychometric properties of the HIT-Q, validation studies have shown the instrument to be reliable and valid (Barriga et al., 2001). Comparable psychometric properties were obtained with different samples in the course of validating the instrument (Barriga, Hawkins, \& Camelia, 2008; Nas, Brugman, \& Koops, 2008; Wallinius, Johansson, Lardén, \& Dernevik, 2011).

The HIT-Q has also been validated in Indian context also. In India, HIT-Q has been validated in adolescent of conflict ridden community of Kashmir (Ara \& Shah, 2015). The goal of the current study was to reassess the psychometric properties of the scores generated by the HIT-16-Q (Ara \& Shah, 2015), in terms of validity and reliability in a large sample of adolescents.

\section{METHOD}

\section{Participants}

The study was conducted in Kashmir Valley of India. The total sample consisted of 1,105 adolescents, of which 544 were male and 561 were female adolescents, aged 16 to 20, selected from different educational institutions.

\section{Measurement}

How I Think Questionnaire (HIT-Q; Barriga et al, 2001): The validated version of HIT Questionnaire (HIT-16-Q; Ara \& Shah, 2015) consisting of 16 items was used in the current study.

Direct and Indirect Aggression Scale (DIAS; Bajorkqvist, Lagerspetz, \& Osterman, 1992) was used to assess the aggressive behaviour. The scale consists of 24 items containing 3 subscales physical aggression (7 items), verbal aggression (5 items) and indirect aggression (12 items), scores ranging from 0 (never) to 4 (always). 


\section{Data analysis}

Statistical analyses were conducted using SPSS and AMOS version 20.0 software packages. To analyze the reliability of the HIT-16-Q scores, Cronbach's Alpha $(\alpha)$ was computed to assess the internal consistency of the HIT-16-Q scores. The construct validity was analyzed using Exploratory Factor Analysis (EFA) and Confirmatory Factor Analysis (CFA). Finally, Convergent validity was assessed by analyzing the correlations of HIT-16-Q scores with constructs that should be related to the measure, such as aggressive behaviours.

\section{RESULTS}

\section{Reliability}

The Cronbach's alpha coefficient for the 16-item HIT-Q scores was obtained. The estimated reliability was found good, alpha of .83, of total HIT-16-Q. The internal consistency of the HIT16-Q was found similar as reported by Ara and Shah (2015) study.

\section{Validity}

To evaluate construct validity, factor analysis was conducted. Prior to the factor analysis, several preliminary analyses were performed to ensure the adequacy of sample size to enable factor analysis. As such, Kaiser-Meyer-Olkine (KMO) and Bartlette's test of sphericity were performed. The sample size is considered adequate if $\mathrm{KMO}$ value is more than 0.50 and Bartlette s test of sphericity is significant if p value is less than 0.05 (Field, 2009). The preliminary analysis of HIT-16-Q was found to be satisfactory. Data was checked for Multicollinearity (Determinant = $.06>.00001)$, revealing no problem. The KMO test $(\mathrm{KMO}=.90>.5)$ verified the sampling adequacy for the analysis. Barttlet's Test of Sphericity, examining whether the R-Matrix resembles the Identity Matrix, was found significant $\left(X^{2}(120)=2445.98, p<.001\right)$, indicated that correlation between items sufficiently large for factor analysis. The values of the Anti-image correlation matrix were above 0.5 for all items (all KMO values $>$.8).

\section{Exploratory Factor Analysis}

Exploratory factor analysis (EFA) was conducted with a principal component analysis (PCA). An initial analysis was run to obtain Eigenvalues for each component in the data. Three components were found having Eigenvalues over Kaiseres Criterion of 1 and in combination explained $42 \%$ (approx.) of variance (see Table 1). The factor loadings of nearly all items were satisfactory.

Different rotations were applied in order to optimize the factor loadings on the extracted components, but couldn't find a better solution. The rotated loading plots (see Figure 3.2b \& 3.2c) were not found better than the unrotated plot (see Figure 3.2a). Evaluating the Scree plot, it suggested a single component, as the difference between $1^{\text {st }}$ component and $2^{\text {nd }}$ component was found large (see Figure 3.1). 
Table 1: Component Matrix showing eigen values and component loadings before rotation

\begin{tabular}{|c|c|c|c|}
\hline Component & 1 & 2 & 3 \\
\hline Eigenvalues & 4.49 & 1.13 & 1.04 \\
\hline 1. It is no use trying to stay out of fights & .44 & & \\
\hline 2. It is ok to tell a lie if someone is dumb enough to fall for it & .54 & & .30 \\
\hline 3. If I really want something, it doesn't matter how I get it & .55 & & -.31 \\
\hline $\begin{array}{l}\text { 4. You should get what you need even if it means someone } \\
\text { has to get hurt }\end{array}$ & .60 & & -.31 \\
\hline 5. You should hurt people first, before they hurt you & .60 & & \\
\hline 6. A lie does not really matter if you don't know that person & .59 & & \\
\hline $\begin{array}{l}\text { 7. You might as well steal. If you don't take it, somebody else } \\
\text { will }\end{array}$ & .58 & -.37 & \\
\hline $\begin{array}{l}\text { 8. If someone is careless enough to lose a wallet, they deserve } \\
\text { to have it stolen }\end{array}$ & .38 & & .43 \\
\hline 9. Everybody breaks the law, it is no big deal & .53 & & \\
\hline 10. Getting what you need is the only important thing & .48 & & -.33 \\
\hline $\begin{array}{l}\text { 11. You might as well steal; people would steal from you if } \\
\text { they had a chance }\end{array}$ & .51 & -.46 & \\
\hline $\begin{array}{l}\text { 12. If people don't cooperate with me, it is not my fault if } \\
\text { someone gets hurt }\end{array}$ & .41 & .41 & \\
\hline $\begin{array}{l}\text { 13. I might as well lie - when I tell the truth, people don't } \\
\text { believe me anyway }\end{array}$ & .50 & & .49 \\
\hline $\begin{array}{l}\text { 14. Sometimes you have to hurt someone if you have a } \\
\text { problem with them }\end{array}$ & .59 & & \\
\hline 15. Everybody steals: you might as well get your share & .56 & -.40 & \\
\hline $\begin{array}{l}\text { 16. If I really want to do something, I don't care if it is legal } \\
\text { or not }\end{array}$ & .55 & .43 & \\
\hline Variance Explained & $28.05 \%$ & $7.04 \%$ & $6.53 \%$ \\
\hline
\end{tabular}



think Questionnaire (HIT-16-Q)

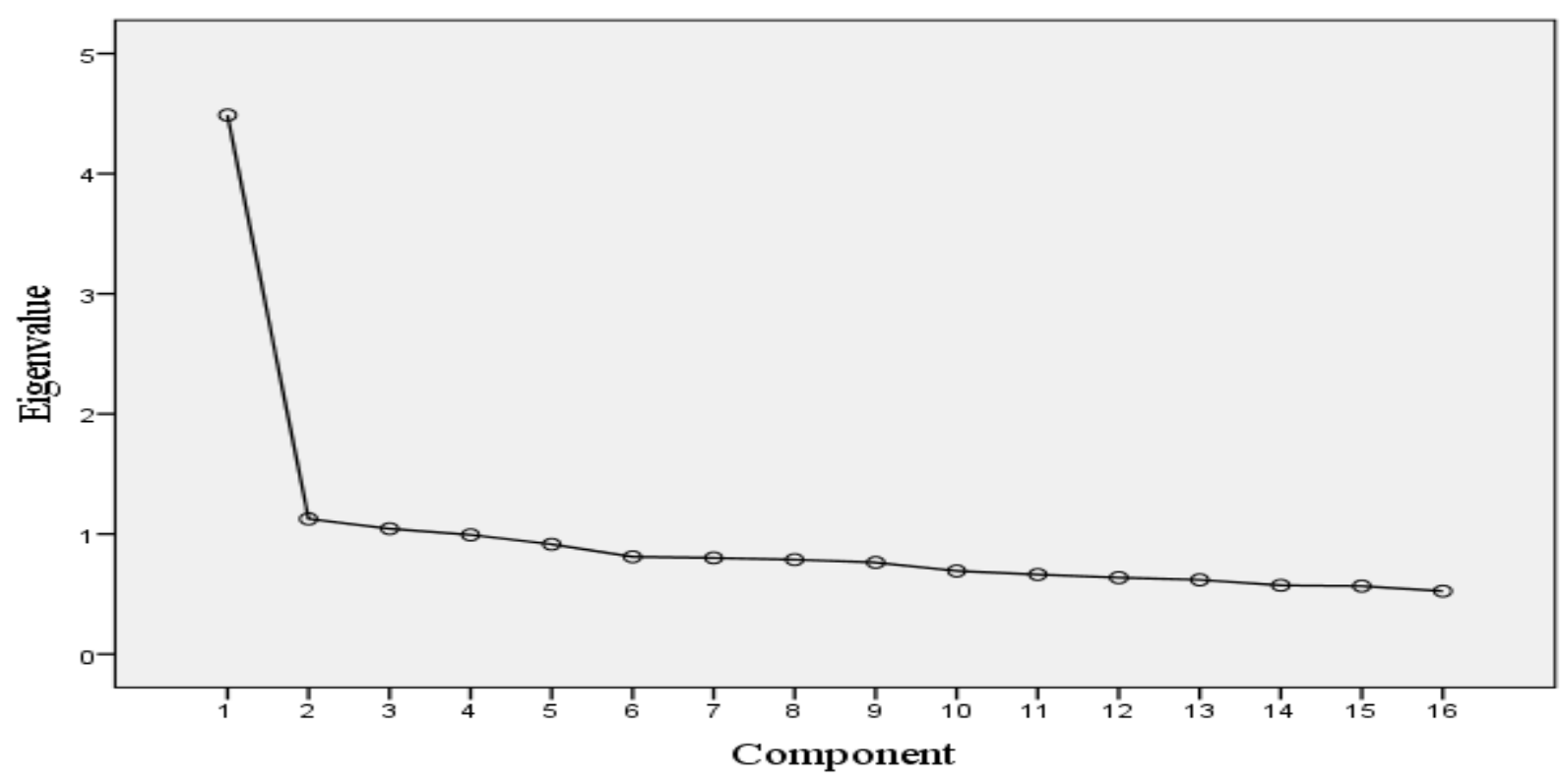

Figure 3.1: Scree Plot

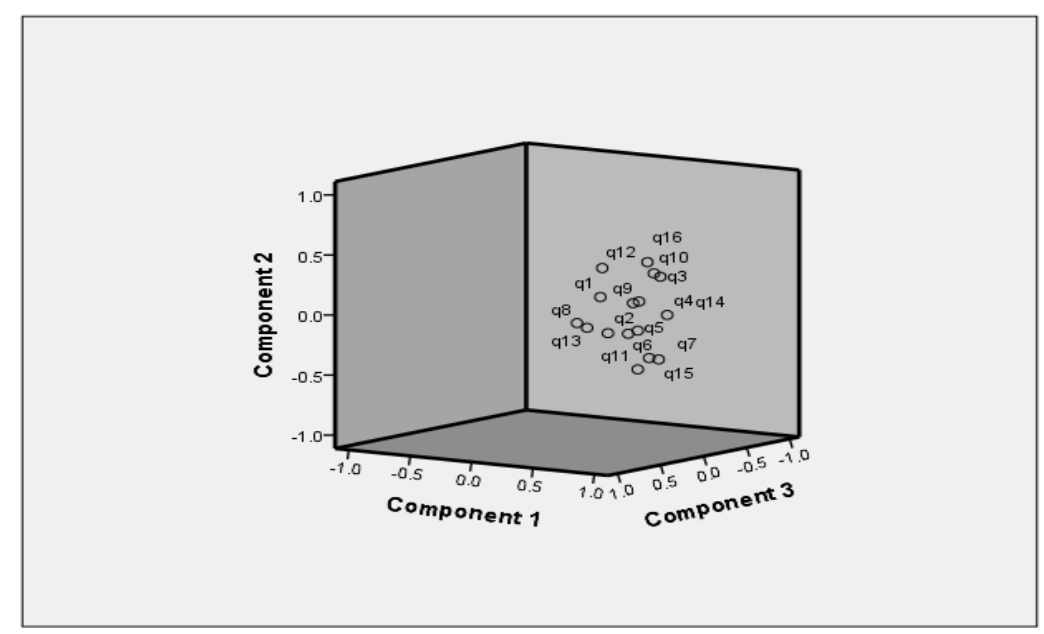

Figure 3.2a: Unrotatated Component Plot 


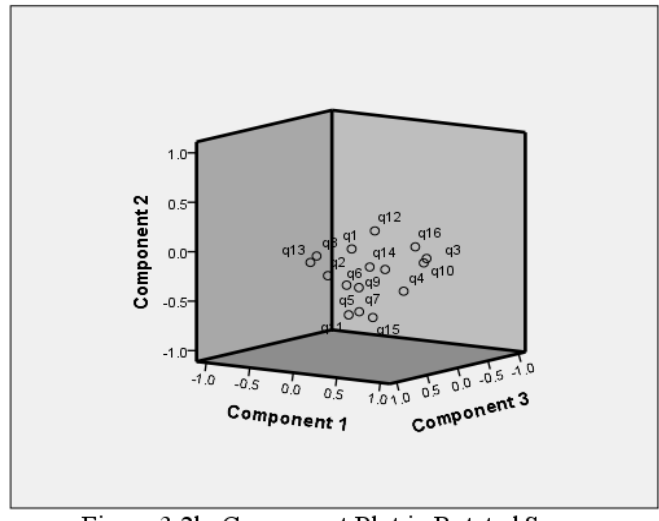

Figure 3.2b: Component Plot in Rotated Space

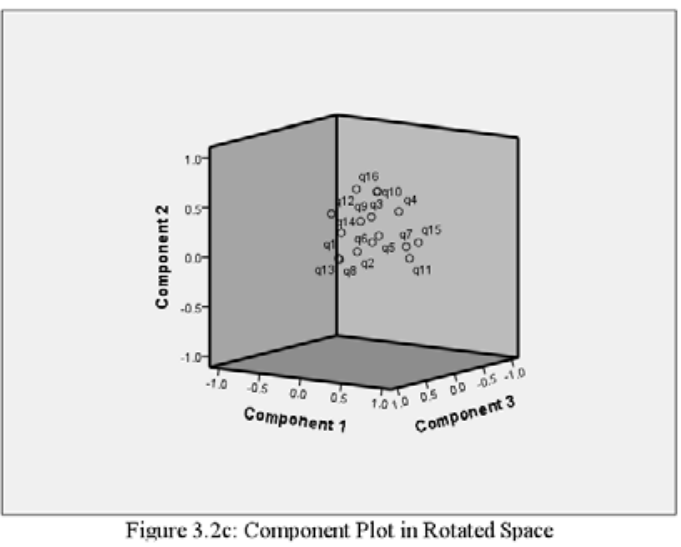

Figure 3.2c: Component Plot in Rotated Space

\section{Confirmatory Factor Analysis}

Confirmatory factor analysis (CFA), using AMOS 20.0, was used to evaluate the adequacy of the unidimensional structural model of cognitive distortions as measured by HIT-16-Q. Model fit may be assessed through a combination of parameter investigations (all parameters should be within acceptable values), the chi-square/ degrees of freedom ratio (which, ideally, should be close to, or less than, two), and various relative fit indices. In this study, we used standard indices and cut-off values to evaluate fit: the Root Means Square Error of Approximations (RMSEA < .08 ), and the Goodness-of-Fit Index (GFI > .90) and Comparative Fit Index (CFI > .90) (see Kline, 1998), as measures of model fit, with all parameters estimated using the maximum likelihood procedure. The model provided an excellent fit to the data, $\chi^{2}(104)=296.90$, Ratio $=$ 2.85 , CFI $=.92, \mathrm{GFI}=.96$, RMSEA $=.05$.

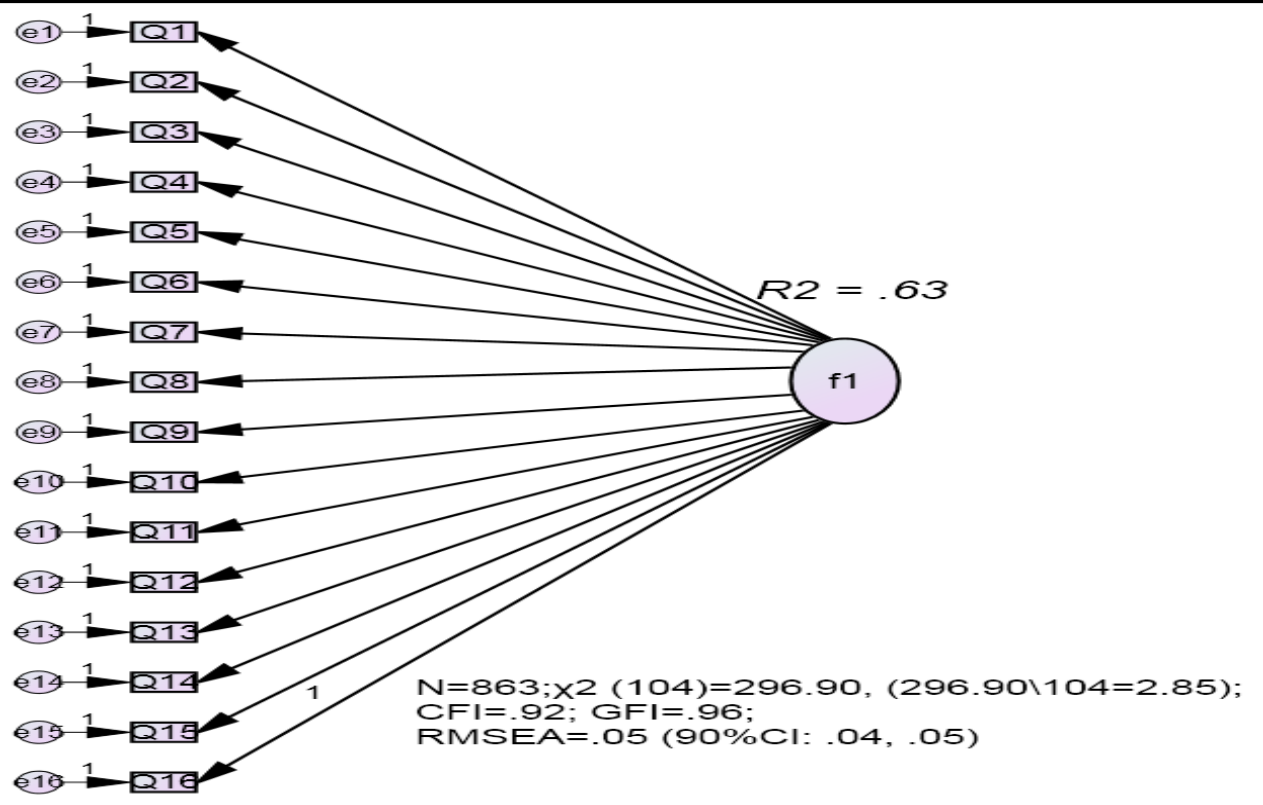

Figure 3.3: Testing Fit for Unidimensional Structural Model of HIT-16-Q 


\section{Correlation between HIT-16-Q and DIAS}

Results on convergent validity are summarized in Table 2. A significant positive correlation emerged between the HIT-16-Q total score and the DIAS score, $r=0.29, \mathrm{p}<0.001$. Accordingly, the adolescents who scored higher on cognitive distortions, scored higher on aggressive behaviours also and vice versa.

Table 2: Correlation between HIT-16 and DIAS

\begin{tabular}{|l|l|l|}
\hline $\begin{array}{l}\text { HIT-16-Q } \\
(\dot{\alpha}=.83)\end{array}$ & $\begin{array}{l}\text { DIAS } \\
(\dot{\alpha}=.89)\end{array}$ & $r=.29 *, \mathrm{n}=242$ \\
\hline$* \mathrm{p}<.001$. & \multicolumn{2}{|l}{} \\
\hline
\end{tabular}

\section{DISCUSSION}

The aim of this study was to assess the psychometric properties of the Indian Validated version of HIT (Barriga, et al., 2001) that is HIT-16-Q (Ara \& Shah, 2015). Where reliability is concerned, it seems satisfactory. The internal consistency of the scale proved good which is similar as obtained in the previous study (Ara \& Shah, 2015). In addition, HIT-16-Q seems valid in that it demonstrated a significant positive correlation to self-reported aggressive behaviours. The present results are similar to those obtained with the original version for which its designers reported a similarly significant correlation between the HIT-Q and self-reported externalizing problems scale, supporting the construct validity of the HIT-Q (Barriga et al., 2001).

Factor analysis provided information on the structure of the HIT-16-Q. The author obtained a three-factor model rather than a four-factor model found in the original structure of the HIT-Q (Barriga et al., 2001). The original structure was made of four cognitive distortion or behavioral referent factors plus one "anomalous responding" and one "prosocial filler". However, the Swedish version also obtained a three-factor solution, one for the criminal mind, one for prosocial behavior and one for social desirability items (Wallinius et al., 2011). Ara and shah (2015) study also reported four factors but with changes in factor-structure. The study revealed that the factor loadings of the items in HIT-16-Q did not correspond to the original domains of HIT-Q. The items loaded highly on different factors, contrary to the findings of Barriga, et al., (2001) validating original HIT-Q, but consistent with some studies revealing different factor structure (e.g., Nas, et al., 2008; Rahim, et al., 2013). The EFA in the current study also could not find better solution, applying different rotations. The Scree plot suggested a unidimensional structure which was further confirmed by the CFA. Thus the findings from the current study also suggest a single one factor for the cognitive distortions as measured by HIT-16-Q.

The findings of the current study are limited to school population. In conclusion, the HIT-Q requires further culturally appropriate revision. Subsequent refinement of the HIT-Q should include evaluation of how culturally relevant the items assessing cognitive distortions are. 


\section{REFERENCES}

Ara, E., \& Shah, S. A. (2015).Validating “How I Think” Questionnaire - Measuring Self-Serving Cognitive Distortions among Adolescents in Kashmir. International Journal of Physical and Social Sciences, 5(6), 117-130.

Bajorkqvist, K., Lagerspetz, M. J., \& Osterman, K., (1992). The direct and indirect aggression scales. Akademic University, Finland.

Barriga, A. Q., Gibbs, J. C., Potter, G. B., \& Liau, A. K. (2001). Test manual for the How I Think Questionnaire. Champaign, IL: Research Press.

Barriga, A. Q., Hawkins, M. A., \& Camelia, C. R. T. (2008). Specificity of cognitive distortions to antisocial behaviours. Criminal Behaviour and Mental Health, 18(2), 104-116. doi:10.1002/cbm.683

Barriga, A. Q., Landau, J. R., Stinson, B. L., Liau, A. K., \& Gibbs, J. C. (2000). Cognitive distortion and problem behaviors in adolescents. Criminal Justice and Behavior, 27(1), 3656. doi: $10.1177 / 0093854800027001003$

Field, A. (2009). Discovering Statistics Using SPSS (3rd ed.). New Delhi, MR: Sage Publications India Pvt. Ltd.

Nas, C. N., Brugman, D., \& Koops, W. (2008). Measuring self-serving cognitive distortions with the "How I Think" Questionnaire. European Journal of Psychological Assessment, 24(3), 181-189. doi: 10.1027/1015-5759.24.3.181

Plante, N., Daigle, M. S., Gaumont, C., Charbonneau, L. Gibbs, J., \& Barriga, A. (2012). Validation of the "How I Think Questionnaire" in a population of French-speaking adolescents with externalizing behaviors. Behav. Sci. Law 30(2), 196-210. doi: 10.1002/bsl.2001

Rahim, K. M., Syariani, M. S. N., Azizah, O., \& Ayu, M. S. G. (2013). Factorial validation of "How I Think" questionnaire among male inmates in Malaysia. Malaysian Journal of Psychiatry, 22(2), 19-3. Available at http:// www.mjpsychiatry.org

Van der Velden, F., Brugman, D., Boom, J., \& Koops, W. (2010). Effects of EQUIP for educators on students' self-serving cognitive distortions, moral judgment, and antisocial behavior. Journal of Research in Character Education, 8, 77-95.

Wallinius, M., Johansson, P., Lardén, M., \& Dernevik, M. (2011). Self-serving cognitive distortions and antisocial behavior among adults and adolescents. Criminal Justice and Behavior, 38(3), 286-301. doi:10.1177/0093854810396139

\section{Acknowledgments}

The author thanks Faheema Aziz Wani, Iram Maqbool Wani, Humaira Gul, and Nighat Qureshi for their contribution to the study. 